\title{
Understanding the Impact of the COVID-19 Pandemic, Lockdowns and Social Isolation on Sleep Quality
}

\author{
David O'Regan (D) ${ }^{1,2}$ \\ Melinda L Jackson (ID) ${ }^{3}$ \\ Allan $\mathrm{H}$ Young $\mathbb{( i D}^{4}$ \\ Ivana Rosenzweig (D) ${ }^{1,5}$
}

'Sleep Disorders Centre, Guy's and St Thomas' Hospital, GSTT NHS, London, UK; ${ }^{2}$ Faculty of Life and Sciences Medicine, King's College London, London, UK; ${ }^{3}$ Turner Institute for Brain and Mental Health, Monash University, Melbourne, Australia; ${ }^{4}$ School of Academic Psychiatry, loPPN, King's College London, London, UK; ${ }^{5}$ Sleep and Brain Plasticity Centre, CNS, loPPN, King's College London, London, UK
Correspondence: Ivana Rosenzweig Sleep and Brain Plasticity Centre, Department of Neuroimaging, Institute of Psychiatry, Psychology and Neuroscience, Box 089, De Crespigny Park, London, SE5 8AF, UK

Email ivana.I.rosenzweig@kcl.ac.uk

\begin{abstract}
The uncertain, ever-changing and an ongoing nature of the COVID-19 pandemic means that it may take some time before we can fully appreciate the negative effect of the pandemic and lockdown on our sleep and mental health. It is increasingly recognised that in the aftermath of pandemic, several persistent sleep, neuropsychiatric and physical sequelae may continue long after the pandemic is over. A body of evidence to date also highlights a significant disparity in sleep and mental health difficulties in specific vulnerable groups in the community, with different temporal profiles and sleep issues that are reported. In this perspective, we argue for a possible mechanistic impact of the COVID-19 pandemic, with its imposed restrictions and social isolation on sleep quality. We similarly discuss some of the potential international differences, as well as similarities, behind reported idiosyncratic biological vulnerabilities that may have contributed to the genesis of sleep issues. Lastly, we propose some possible implementations and innovations that may be needed in restructuring of sleep disorders services in order to benefit recovering COVID-19 patients.
\end{abstract}

Keywords: sleep, insomnia, hypersomnia, lockdown, COVID-19 pandemic, social isolation

\section{Introduction}

During the ongoing COVID-19 pandemic across the globe, public health measures have been implemented to reduce community transmission of the virus, including stay-athome orders, quarantine, and social distancing measures. ${ }^{1}$ These measures have had a profound economic and social impact, including disruption to employment and education. ${ }^{1,2}$ Moreover, these restrictions have also had important neuropsychiatric consequences for the population as a whole, with forced social isolation leading to an increase in reported psychiatric symptoms, including stress, anxiety and depression for all individuals. ${ }^{1-4}$ It has been suggested that this relationship seems to be moderated by the loneliness associated with prolonged periods of lockdown, and maladaptive changes in lifestyle, including disturbed sleep. ${ }^{1-3}$ The notion that such a global event would lead to a further global pandemic of disturbed sleep in wide swathes of the population is perhaps unsurprising, given that sleep is pivotal for recovery and physiologic adaptation to the waking experiences. ${ }^{5}$ The bidirectional relationship between sleep and emotional brain function appears to be supported by the findings of studies that build on longstanding clinical observations of co-occurring mood and sleep disorders. ${ }^{6,7}$

Over the last two years, impressive global scientific effort has been undertaken to examine the clinical sequelae and biology of the severe acute respiratory 
syndrome coronavirus 2 (SARS-CoV-2) virus, with increasing awareness that SARS-CoV-2 can affect the central and peripheral nervous system. ${ }^{2,8}$ In a recent metaanalytical estimate of point prevalence of neurological or neuropsychiatric symptoms, sleep disorders were recognized as one of the most frequently reported symptoms, with a 23.5 -point prevalence, ${ }^{2}$ similar to depression and headache estimates. ${ }^{2}$

The heterogeneity of reported neuropsychiatric symptoms precludes us from drawing conclusions about the potential differential mechanistic platforms behind the detrimental biological effects of isolation and heightened stress versus those potentially solely exacerbated by the SARS-CoV-2 infection. The resulting effects and subsequent sleep issues in both cases are most likely due to a complex interplay of a myriad of biological and environmental factors, including: electrolyte abnormalities, liver inflammation, impaired renal function, impaired oxygenation, hyper-inflammation, and isolation due to public health concerns., ${ }^{4,9}$ Moreover, current evidence suggests that idiosyncratic factors, including, for example, pre-morbid psychiatric illness, may be both a risk factor for and a consequence of COVID-19. ${ }^{1,4}$ In a pre-COVID-19 study that analyzed sleep data from 41,094 participants, ${ }^{10}$ socioeconomic disadvantage, ethnic minority background, shift work, unhealthy lifestyle, poor health, depressive symptoms and obesity were highlighted as the major risk factors associated with poor sleep. ${ }^{10}$ It has also been suggested that around a third of the total effect of socio-economic deprivation on poor sleep may be mediated through depressive symptoms. ${ }^{10}$

Thus, vulnerable individuals appear to be at greatest risk of developing sleep problems due to these multiple contributors, and may experience both acute and long-term sleep and other neuropsychiatric disorders. ${ }^{1,4}$ In this perspective review, we draw on evidence from various lines of ongoing research to assess how the impact of the COVID-19 pandemic, imposed restrictions and social isolation have impacted sleep quality. We evaluate how different countries and particular conditions, including idiosyncratic biological vulnerabilities, have contributed to the genesis of sleep issues, and we consider possible implementations and innovations needed in restructuring of sleep disorder services to benefit recovering COVID-19 patients.

\section{Sleep and Neuropsychiatric Complications of the COVID-I 9 Pandemic, Lockdown, Infection and Vaccination COVID-19 Pandemic and Lockdown}

One of the earliest studies of the effects of COVID-19 pandemic reported findings from a survey of 2254 participants, aged 16-75 years. ${ }^{11}$ The findings echoed a general mood pervasive in the United Kingdom (UK) in May 2020, just a few months after the UK government announced lockdown measures. ${ }^{11}$ This survey for the first time suggested a possible significant short (and long) term impact of the COVID-19 pandemic on the quality of sleep and anxiety levels in the UK. ${ }^{11}$ In the survey, six out of ten people reported suffering from disturbed, unrefreshing sleep, of both shorter and longer duration, with unusually vivid dreams. ${ }^{11}$ A slightly later UK cross-sectional online surveybased study on adults living through the COVID-19 pandemic similarly found that $69.4 \%$ of 843 participants had disturbed sleep patterns. ${ }^{12}$ Of those, less than half (44.7\%) had refreshing sleep, $45.6 \%$ were sleepier than before the lockdown and $65.2 \%$ reported an impact on their mental health, with $25.9 \%$ reporting drinking more alcohol during the lockdown. ${ }^{12}$ Of note is that respondents with suspected COVID-19 were also found to have more vivid nightmares and abnormal sleep rhythms. ${ }^{12}$ These findings were mirrored by an Italian online web-based study of 5988 adults, also undertaken during lockdown, which revealed that disturbed sleep patterns in part predicted higher dream recall, as well as nightmares. ${ }^{13}$ Other important psycho-social predictors of nightmares in this study included: anxiety, depression, those who had stopped working, and those who had friends/relatives who were infected by or who had died of COVID-19 ${ }^{13}$. As discussed further below, when taken together, these findings are in keeping with the continuity hypothesis of dreaming. ${ }^{14,15}$

Very early on, we, and others, argued that some of those reported sleep symptoms (eg vivid dreams) possibly reflected the biological response to the extraordinary stress and uncertainty that the populace was facing. ${ }^{1,16-19}$ For example, it is known that rapid eye movement (REM) sleep structure, during which most of our dreaming occurs, changes first during chronic stress, and more so than any other measured sleep characteristic. ${ }^{5}$ Moreover, REM sleep has been suggested to play a role in recalibrating the sensitivity and specificity of the brain's response to 
emotional events on both ends of positive and negative spectrum, suggesting a bidirectional impact of any such REM sleep disturbance on affected individuals. ${ }^{6,20}$ Similarly, Steenblock et al argued for a link between the ongoing SARS-COV-2 outbreak and the neuroendocrine stress axis. ${ }^{19}$ They warned of uncertain long-term outcomes for those who have been actually infected, as well as for those who are not necessarily infected themselves, but who may suffer lasting consequences as a result of loss, grief, isolation, deprivation, and other psychological outcomes of ongoing quarantine or shelter in place measures. ${ }^{19}$ These groups include health care workers, and others whose lives were severely affected. ${ }^{19}$ From earlier coronavirus outbreaks, it has been evident that survivors and health care workers are at increased risk of developing mental illness years after the outbreak. ${ }^{19}$ It has been argued that such long-lasting impacts might be due to morphological changes in the brain and in the organs of the hypothalamic-pituitary-adrenal (HPA) axis, ${ }^{19}$ with the added complexity of widely differing gender responses to stress. Worryingly, it has also been suggested that permanent epigenetic changes in stem cells may occur due to actual infection, as well as that they may result from the different forms of severe stress, ${ }^{19}$ further suggesting likely profound and long-term effects of the current pandemic.

\section{COVID-19 Infection}

Studies to date have also suggested that microvascular infarcts and haemorrhages, which are part of the systemic coagulopathy and vasculopathy of COVID-19, may be critical in the development of encephalopathy, delirium and other neurological and psychiatric manifestations of SARSCoV-2 infection. ${ }^{9}$ The consensus on whether disturbances of higher mental function occur predominantly without direct infection of the central nervous system neurons has not yet been reached; however, it does appear that the virus can enter the brain, ${ }^{9}$ when it may infect vascular and immune cells. ${ }^{9}$ The direct SARS-CoV-2 invasion of the CNS likely occurs at the blood-brain barrier via transcellular migration through host endothelial cells, paracellular migration through tight junctions, and an immune system "Trojan horse" cell passing through the blood-brain barrier. ${ }^{4,9}$

Our group has recently proposed that a relatively unique fingerprint of sleep and ultradian rhythms disturbance, along with heightened levels of anxiety, during SARS-CoV-2 infection may in some patients result from viral binding to neuronal angiotensin-converting-enzyme-2 (ACE2)receptors in a specific part of the brain's hypothalamic paraventricular nucleus (PVN) (Figure 1). ${ }^{18}$ It is known that SARS-CoV-2 can directly decrease ACE2 activity and lead to cell toxicity in other tissues. ${ }^{9,18}$ This interaction is believed to subsequently lead to a myriad of symptoms, from increased blood pressure to kidney failure. ${ }^{18,21}$ Arguably, any such potential direct or indirect SARS-CoV-2 effect on the PVN circuitry, a major brain computational hub that ensures timely processing of multiple physiologic signals, would significantly affect pivotal short-term biological rhythms and sleep patterns, resulting in increased, debilitating and misdirected anxiety. ${ }^{18}$

Unsurprisingly, the data regarding the long-term cognitive consequences of COVID-19 is still lacking, but one study of 279 patients hospitalized with COVID-19 found that, three months after discharge, $34 \%$ reported significant memory loss and $28 \%$ described impaired concentration., ${ }^{4,22}$ Comparable findings have been observed following infection with other coronaviruses, in which $20 \%$ reported cognitive deficits months to years after initial infection. ${ }^{2}$ In keeping with this, Taquet et al, ${ }^{23}$ who have recently reported results from a large USA dataset, showed that in the six months after SARS-CoV-2 infection, about a third of these individuals had a neurological or psychiatric disorder, more than comparative figures for influenza. ${ }^{23}$ Most of these heterogenic neurological or psychiatric disorders were more common in patients who had COVID-19 than in those who had influenza and those who had other respiratory tract infections. ${ }^{23}$ Another study that examined the differential psychological impact of COVID-19 infection in 7917 participants, of whom 49.7 were healthcare professionals with a high representation of female participants, ${ }^{24}$ reported suicidal thoughts in $32 \%$ of respondents. ${ }^{24}$ Healthcare professionals reported mild depression and anxiety in higher proportions to other respondents. ${ }^{24}$ Increasing age and female gender were linked to higher compliance with governmental advice on COVID19 , whereas higher education, homeowners, key worker status, high alcohol, drug use and participants with pre-existing suicidal thoughts were linked to lower compliance with governmental advice. ${ }^{24}$ Moreover, a striking psychological impact on the wellbeing of frontline healthcare professionals was demonstrated, with many clinicians increasingly raising concerns about future retainment as well as recruitment to medical specialty and healthcare sector. ${ }^{24}$

\section{COVID- 19 Vaccination}

Finally, another potentially controversial side to the ongoing pandemic is the possible unknown long-term effect of different COVID-19 vaccines, for which the 


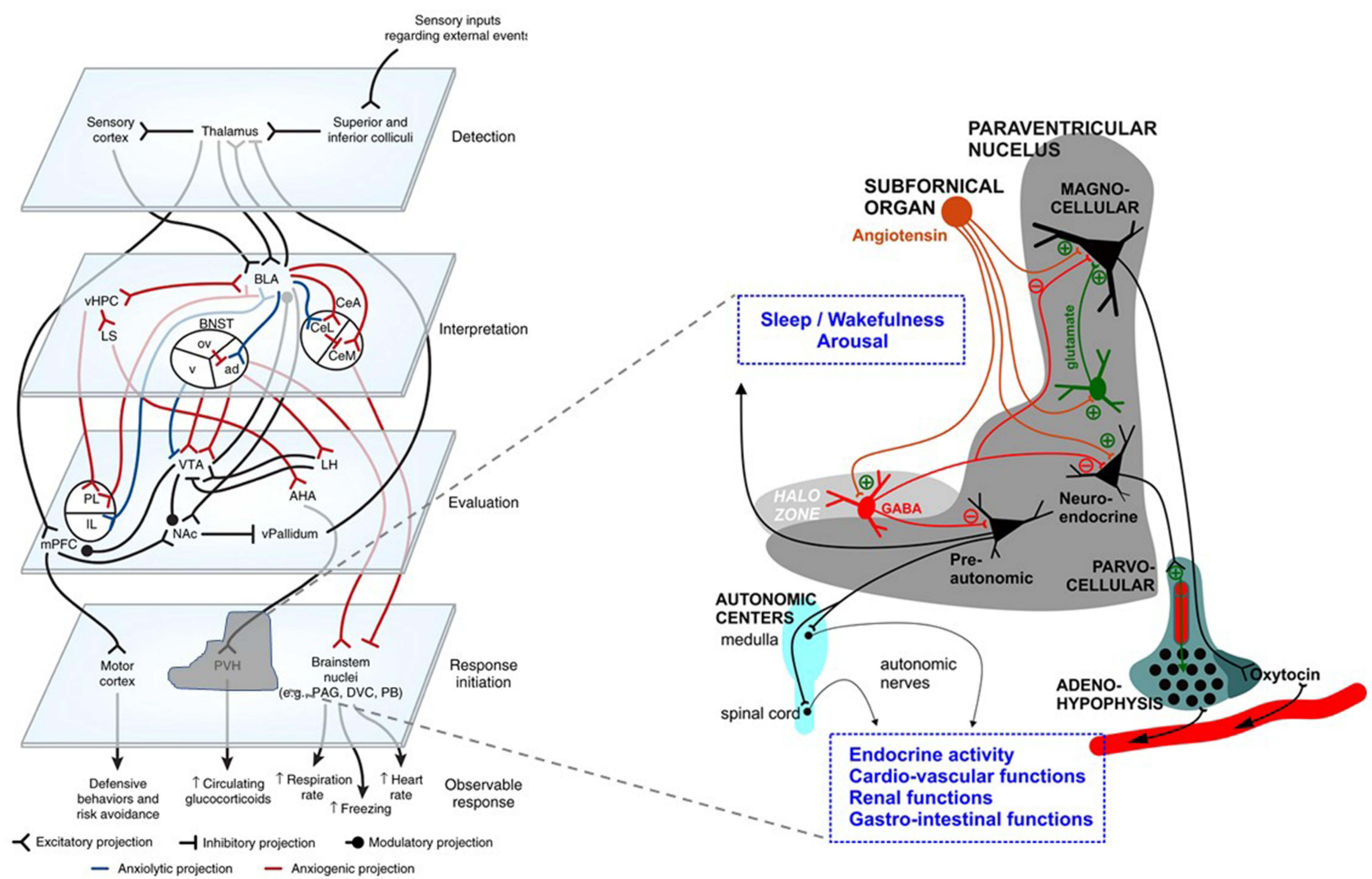

Figure I The schematic illustration of the basic neuronal organization of the paraventricular nucleus (PVN) of the hypothalamus depicting its purported role in a four-step model of neurocircuitry of anxiety. On the left, a four-step model by Calhoon and Tye is shown: according to this model external events are detected, interpreted, evaluated, and responded to by succeeding levels of highly interconnected neural circuits. Reproduced by permission from Springer Nature, Nat Neurosci, Resolving the neural circuits of anxiety, Calhoon GG, Tye KM. 2015;18(10):1394-1404. Copyright @ 2015, Nature Publishing Group, a division of Macmillan Publishers Limited. ${ }^{92}$ Events are further interpreted as threatening or nonthreatening depending on the balance between opposing circuits; when the balanced is shifted toward projections interpreting events as threatening, this leads to anxiety. On the right, PVN's neurocircuitry is depicted. Afferent inputs to the nucleus arrive from many important integrative centers of the medulla, pons and hypothalamus. Subfornical organ is among major inputs with identified angiotensin release acting directly on magnocellular and parvocellular neurons, or indirectly via intranuclear circuitry that includes inhibitory Gamma-aminobutyric-acid (GABA) (red) and excitatory glutamate (green) interneurons. Changes within paraventricular angiotensin subcircuitries are described under stress condition and are reflected on release of stress hormone and arousal. GABA interneurons have been shown to express angiotensin-converting-enzyme-2 (ACE2), ${ }^{93}$ and as such might be targeted by severe-acute-respiratory-syndrome-coronavirus-2 (SARS-CoV-2). The majority of GABA interneurons have been localized to the halo zone surrounding the PVN, and their role as an additional gatekeeper and integrator in controlling the excitability of PVN outputs has been proposed. ${ }^{94}$ Any changes in the PVN circuitries, due to their major control over most of neuro-endocrine axes and neuronal autonomic centers may cause robust alteration in homeostatic regulation, and through influence on regulatory brain centers impact on sleep and wakefulness, increased propensity to trigeminal autonomic cephalalgias and alteration of ultradian rhythms.

Notes: Reproduced from Rosenzweig I, Mitrečić D, Petanjek Z, et al. Does damage to hypothalamic paraventricular nucleus underlie symptoms of ultradian rhythm disorder and an increased anxiety in coronavirus disease 20I9? Croatian Medical Journal. 2020;6I(4):377-380. Copyright (C) 2020 by the Croatian Medical Journal. All rights are reserved. Creative Commons Attribution License ${ }^{18}$.

Abbreviations: ad, anterodorsal nucleus of the BNST; ACE2, angiotensin-converting-enzyme-2; AHA, anterior hypothalamic area; BLA, basolateral amygdala; BNST, bed nucleus of the stria terminalis; CeA, central amygdala; CeL, lateral subdivision of the central amygdala; CeM, centromedial subdivision of the amygdala; DVC, dorsal vagal complex; GABA, Gamma-aminobutyric-acid; IL, infralimbic division of the mPFC; LH, lateral hypothalamus; LS, lateral septum; mPFC, medial prefrontal cortex; NAc, nucleus accumbens; ov, oval nucleus of the BNST; PAG, periaqueductal gray; PB, parabrachial nucleus; PL, prelimbic division of the mPFC; PVH/PVN, paraventricular nucleus of the hypothalamus; SARS-CoV-2, severe-acute-respiratory-syndrome-coronavirus-2; v, ventral BNST; vHPC, ventral hippocampus; vPallidum, ventral pallidum; VTA, ventral tegmental area.

SARS-CoV-2 spike protein was a major target. ${ }^{21}$ It is clear that the development of SARS-CoV-2 spike protein-based COVID-19 vaccine has been one of the major scientific and medical breakthroughs of the 21 st century, which has provided much-needed hope on how to end the current pandemic. However, the limited findings of several in vitro and post-mortem studies suggest that the recombinant SARS-CoV-2 spike protein S1 subunit could be potentially sufficient to promote cell signaling without the remaining viral components. ${ }^{21,25,26}$ Similarly, at present, the insertional mutagenesis safety of mRNA-based vaccines remains largely unresolved. ${ }^{27}$ Going forward, it will be pivotal to keep evaluating the data from SARS-CoV-2 infected individuals, as well as from the healthy individuals who received the spike protein-based vaccines, with further stringent investigations of the effects of the SARS- 
CoV-2 spike protein in appropriate animal models. ${ }^{21}$ Taken together, the data to date strongly suggest that monitoring of all affected populations for an extended time following the current pandemic might be needed. ${ }^{19}$

\section{The Effects of Pandemic and Lockdown on Sleep, Circadian Timing and Mental Health Across the Globe}

The COVID-19 pandemic and its associated lockdown(s) have evidently resulted in restrictive lifestyle changes, ${ }^{28}$ with alterations in important zeitgebers, such as light exposure, meals, social interactions, and exercise resulting in further disruption of chronobiological rhythms. ${ }^{29}$ Confinement to homes, which doubled as new workplaces, with simultaneous increase in responsibilities, such as home-schooling ${ }^{29}$ additionally occurred in an anxious milieu, with fears regarding contracting COVID-19, employment security, finances, and concerns about the welfare of loved ones. ${ }^{30}$ Many of these anxieties were further fueled by a constant deluge of ever-changing news and government information, leading some to nickname the crisis "an information pandemic". 31,32 The concomitant impact of these lifestyle changes has provided the perfect storm to adversely impact sleep quality, by individually affecting each of the three sleep regulatory processes: the homeostatic sleep drive, the circadian rhythm, and the arousal system. ${ }^{33}$

During the very early days of the pandemic, in the early part of 2020, an interesting dichotomy with regard to reported changes in sleep was noted around the globe. Studies from Europe, US and UK have reported an increase in sleep opportunity, sleep duration and reduced "social jetlag" during the pandemic. ${ }^{11,12,34-37}$ Such positive changes are most likely due to alterations in pressurized social and environmental factors, including changes in employment practices, such as remote and flexible working. ${ }^{38,39}$ Smartphone data of 2.9 million nights of sleep recordings taken from participants across different continents indicated a sudden increase in sleep duration during the early stages of the pandemic, compared to the same time a year earlier. ${ }^{36}$ In addition to increases in sleep duration, changes to sleep timing have also been observed. In one global survey, nearly three quarters of respondents indicated that their sleep patterns have changed as a result of lockdowns, with the majority indicating that their sleep and wake times were delayed compared to before the pandemic, and $43 \%$ of respondents indicating that their current sleep timing was more in line with their body clock. $^{40}$ Increasing flexibility in sleep-wake routines resulting from stay-at-home orders has clearly been of benefit to many in society who would have otherwise suffered with reduced sleep opportunity due to occupational and lifestyle commitments. However, for others, being afforded greater flexibility in sleep timing appears to have resulted in more irregular sleep-wake timing, which is known to have adverse effect on academic performance and health outcomes. ${ }^{41,42}$ Indeed, despite some initial benefits that were reported in some sections of western society as the pandemic progressed, the majority of populace has overwhelmingly described disturbed sleep patterns, and diminished sleep quality in comparison to pre-pandemic sleep patterns across the globe. ${ }^{34,35,37,40,43}$

In one global survey, $73 \%$ of respondents reported poor sleep quality, scoring more than five on the Pittsburgh Sleep Quality Index (PSQI), ${ }^{40}$ with around $57 \%$ of respondents indicating that their sleep was worse currently compared to before the pandemic. ${ }^{40}$ This is in line with other prevalence estimates of poor sleep of around 50-55\% from Italy, $^{40,43}$ however much lower levels were reported around the same time in China (36\%). ${ }^{44}$ These differing rates are likely due to the predominantly female respondents in some surveys, potentially amplifying the prevalence rates of poor sleep. This highlights the limitations of rapid data collection of convenience samples, which may not be representative of the population. Importantly, poor sleep quality has been linked to poorer mental health, both pre-pandemic and in the recent pandemic literature. ${ }^{40,45}$ In the same global study, it has been shown that individuals who scored more than eight points on the PSQI had a three-fold increased prevalence of significant anxiety, stress and depressive symptoms, even when controlling for pandemic-related factors and demographics. ${ }^{40}$ While further longitudinal studies are needed to uncover the directionality of these associations, it would appear that addressing poor sleep during lockdowns may help to mitigate some of the adverse mental health effects.

Moreover, for those who were home confined during lockdown, circadian rhythm disruption, ${ }^{29}$ compounded by increased digital screen time $e^{46,47}$ has adversely affected sleep timing and quality. ${ }^{30,48}$ Increased daytime napping, also seen during home confinement, has been shown to reduce sleep pressure (ie the homeostatic sleep drive), resulting in delayed bedtimes, and prolonged sleep onset latency. ${ }^{30,49}$ Arguably, later bedtimes can also adversely 
impact slow wave sleep, and its purported function of enabling the glymphatics clearance of brain toxins, ${ }^{50}$ as well as impact on the overall sleep quality. ${ }^{51}$ Thus, circadian and homeostatic factors that interact to regulate sleep have all been adversely affected by lockdown.

When exploring differences between countries in terms of the rates of sleep difficulties or mental health symptoms, little difference emerged despite dramatic differences in infection rates and COVID-19 related deaths across countries ${ }^{1,11,12,17,35,37,52}$ (also see Figure 2). ${ }^{1}$ Respondents from countries with high case numbers did report higher stress levels, although the effect size was small. ${ }^{40}$ An interesting case study by Czeisler et al explored the impact on sleep and mental health in respondents from Victoria, Australia - a city with one of the longest and most stringent lockdowns globally (112 days) with relatively low SARSCoV-2 case numbers $(0.32 \%) .{ }^{53}$ Compared to the status before the pandemic, during Stage 4 restrictions in September 2020 participants reported spending more time in bed and having more trouble sleeping, with one third of respondents reporting anxiety or depressive symptoms and one in ten reporting suicide ideation occurring over the last 30 days. ${ }^{53}$ Around $21 \%$ of respondents reported insomnia symptoms, slightly higher than that reported in April 2020
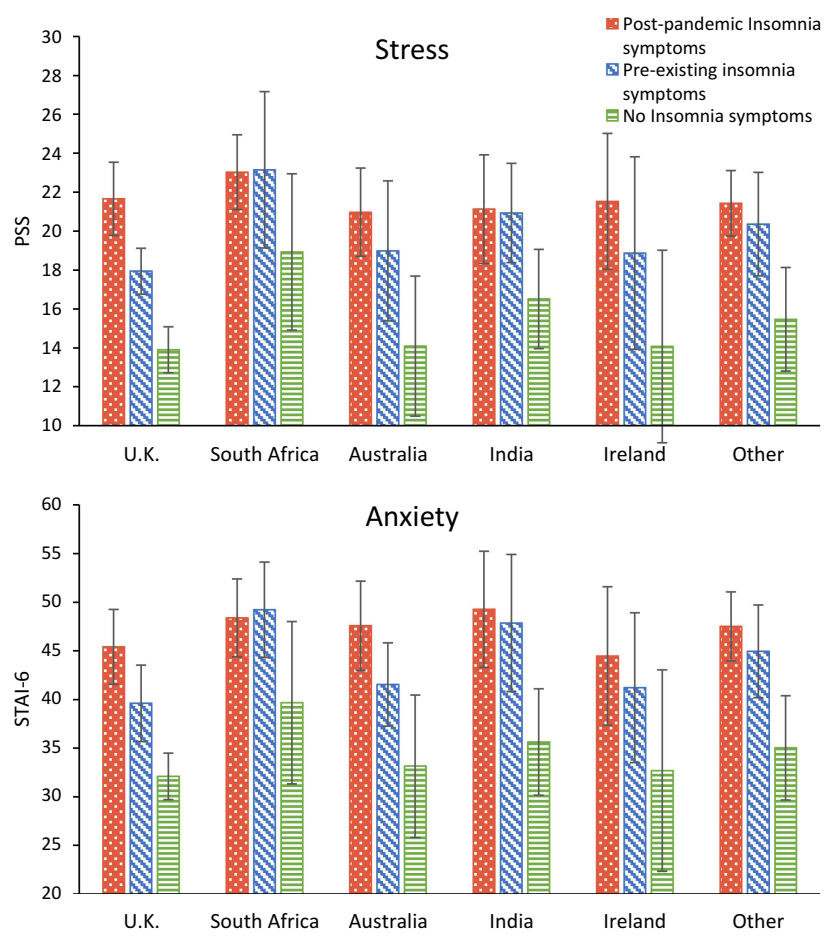

near the start of lockdown. ${ }^{53}$ Critically, suicide ideation was 1.86-fold more prevalent in those with insomnia symptoms. ${ }^{36}$ Victorians, who endorsed higher mental health symptoms, had a shorter sleep duration ( $<$ six hours), spent more time in bed, had more trouble falling asleep and reported maintaining a less regular sleep-wake schedule. ${ }^{53}$ On a positive note, those who adapted their behavior and were able to keep more regular sleep times and avoid COVID-19 media coverage had a lower prevalence of depression and anxiety symptoms. ${ }^{53}$ This study provided a unique perspective on the impact of lockdown on sleep and mental health symptoms, suggesting that these effects may not transient, as well as that individual idiosyncratic factors might play a significant role in the valence of the reported outcomes. ${ }^{53}$

\section{Mediating Factors and Vulnerable Groups}

A body of evidence to date similarly highlights a significant disparity in sleep and mental health difficulties in specific groups in the community. For instance, it has been reported that in South Africa, the lockdown has caused substantial economic hardship, especially among poor and vulnerable people, with possible important long-term consequences. ${ }^{54}$ Moreover, restrictions on movement and the risk of
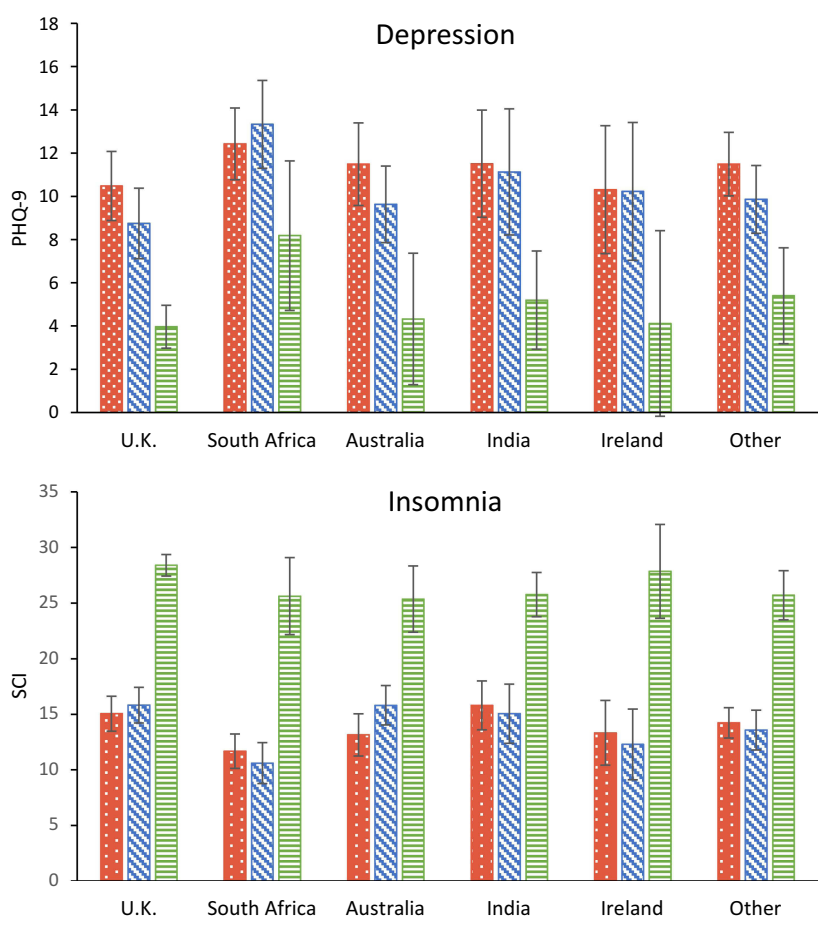

Figure 2 Stress, depression, anxiety, and insomnia symptoms across countries with the top five number of respondents by insomnia group $(\mathrm{n}=2525)$. Error bars indicate $95 \%$ confidence intervals.

Abbreviations: PSS, perceived stress scale; STAI-6, 6-item state anxiety inventory; PHQ-9, patient health questionnaire-9 item; SCl, sleep condition indicator. 
contracting COVID-19 in health care facilities in South Africa have brought major reductions in the use of health services, thereby compromising continuity of care for people with HIV, tuberculosis, and chronic noncommunicable diseases. ${ }^{54}$ The COVID-19 pandemic has also had a particularly significant impact on people with Alzheimer's disease (AD) and other dementias internationally. ${ }^{3}$ This vulnerable population has been shown to be particularly susceptible not just to SARSCoV-2 infection, but also to the negative effects of the measures taken worldwide to control the spread of the virus, with loneliness and isolation aggravating and accelerating the clinical picture. ${ }^{3}$

Internationally, an overwhelming trend towards later bedtimes, delayed sleep onset, a reduction in total sleep time and quality, has been reported together with increased daytime napping during lockdowns. ${ }^{30,49}$ Loneliness, uncertainty, depression and COVID-19 related-worries were only some of the important psycho-social drivers in underlying these changes. ${ }^{49}$ These changes were more pronounced in younger people, women, those with a pre-existing neurologic and psychiatric conditions, unpaid caregivers and frontline workers, those who were unemployed, and in those who lived in less supportive welfare states,${ }^{55}$ suggesting that these groups may be more vulnerable to the acute and longer term effects of the pandemic. ${ }^{40,56}$ Younger adults (under 45 years) appeared to be more affected during the pandemic, and higher levels of stress, anxiety and depressive symptoms were reported in that age group by comparison to older age groups. ${ }^{40,56,57}$ In addition, loneliness and financial difficulties have been linked to the higher rates of mental health symptoms in this age group. ${ }^{40}$

Reflecting the pre-pandemic sex disparity in insomnia prevalence, women increasingly appear to be more affected by lockdown in terms of their sleep. In a longitudinal study in Italy, insomnia severity and mental health symptoms were reported to be greater in women than men during the early stages of lockdown, ${ }^{58}$ and this finding closely echoes other reports across the globe. ${ }^{40,49,52}$ Interestingly, however, in follow-up surveys and as the lockdown continued, the gender-gap narrowed across all measures. ${ }^{58}$ Thus, the impact of prolonged lockdown may have differential temporal effects on men and women, which may necessitate differential therapeutic approaches.

Historically and during the pandemic, poor sleep has been linked to greater financial difficulties, greater disability, and an increase in alcohol consumption. ${ }^{40}$ Behavioral factors are also likely to directly impact sleep during lockdowns. For instance, a decrease in sunlight exposure, daily exercise and increased phone usage before bedtime have been linked to poorer sleep quality during lockdown. ${ }^{40}$ The interactions between sleep, diet, substance use and exercise are complex, with changes in one domain likely to impact the others. ${ }^{59}$ For example, we know that inadequate sleep is likely to promote poorer health behaviors, such as reducing the motivation to exercise and to eat healthily. ${ }^{60}$ Moreover, those with poorer sleep during lockdown may be tempted to self-medicate with alcohol $^{61}$ and/or other illicit substances, ${ }^{62}$ in hopes of improving their sleep. Unfortunately, such strategies merely worsen sleep, and further accelerate or initiate insomnia. ${ }^{63}$ An association between alcohol abuse and the risk of violent offending has previously also been shown, with particular relevance for vulnerable sections of the society, including those with premorbid psychiatric disorders. ${ }^{64}$

Many of these health behaviors have been shown to be affected by the psychological status and social connectedness of the individual, both of which have been adversely affected by lockdown. ${ }^{65,66}$ For many, lonely days mean lonely nights, resulting in poor sleep quality, with subsequent adverse effects on other health behaviors. ${ }^{67}$ Social isolation can potentially also lead to a precarious state of chronic heightened anxiety that can increase individual's vulnerability to coercion and abuse during the pandemic. ${ }^{68}$ Indeed, poorer sleep quality and higher levels of loneliness have been found to mediate the relationship between stress and mental health symptoms (depression, anxiety), highlighting the critical, but modifiable, impact of sleep on mental health during lockdown. ${ }^{40}$

\section{Pragmatic Focus on Sequelae of Insomnia and Hypersomnia}

Insomnia symptoms frequently manifest in the face of acute life changes or stressors, such as pandemics, ${ }^{69}$ and unsurprisingly, studies conducted during the COVID-19 pandemic have overwhelmingly and consistently shown a significant increase in insomnia symptoms, ranging from $18 \%$ to $63 \%$. ${ }^{16,37,70,71}$ Whilst some of these insomnia symptoms may be categorized as mild, it has been well accepted that transient sleep disturbances can later lead to chronic insomnia disorders. ${ }^{33}$ A recent study by Meaklim et al reported on difference in mental health symptoms in individuals with pre-existing insomnia, individuals who had developed insomnia symptoms during the pandemic and healthy sleepers. ${ }^{1}$ For individuals with post-pandemic insomnia symptoms, $77 \%$ believed that factors 
surrounding the pandemic triggered their insomnia symptoms. ${ }^{1}$ Those who developed insomnia symptoms during the pandemic also had higher levels of stress, anxiety and depressive symptoms compared to individuals with pre-existing insomnia symptoms, or compared to those who were sleeping well (Figure 2). ${ }^{1}$

Paradoxically, 25-28\% of individuals with pre-existing insomnia disorder reported clinically meaningful improvements in sleep quality during the pandemic, ${ }^{1,72}$ suggesting that changes in sleep in response to the stressor of the pandemic are not uniform across individuals. While the reasons for this are likely to be multi-faceted, reduction of social and occupational pressure and attenuation of negative mood states have been proposed as potential mechanisms. ${ }^{1}$ Moreover, individual experiences of lockdown can vary considerably, and for some people with insomnia, lockdown restrictions may have created a beneficial environment, reducing the stress of work and social commitments. Indeed, a qualitative synthesis of personal experiences during lockdown revealed that those with better sleep quality used more positive language, indicative of social support and positive well-being (eg "friend"). ${ }^{40}$ Some clinicians have argued that enhancement of social support, and an increased social cohesion in some societies during lockdown might have helped to buffer predominantly negative impacts of the pandemic on sleep and well-being. ${ }^{1}$

A meta-analysis of 177 published studies revealed that insomnia symptoms were most prevalent in healthcare professionals $(43 \%)$, which further indicated that age, gender, country and marital status did not impact prevalence rates. ${ }^{71}$ As already mentioned, health professionals, especially frontline workers who regularly care for COVID-19 infected patients, may be at higher risk of insomnia, because of increased fear of risk of infection and death, as well as irregular and more frequent work schedules. $^{73-75}$ For other "essential" workers besides healthcare, earlier wake times, and reduced total sleep times were also seen, when compared to "non-essential" workers. $^{17,76}$

There is a paucity of published research regarding lockdown and hypersomnolence, although the data from surveys around the globe clearly suggest that decreased sleep quality has been accompanied by both the decreases, and increases in sleep duration during the pandemic. ${ }^{11,12}$ Historically, hypersomnolence has been seen as a direct consequence of viral infection and/or treatment in pandemics; ${ }^{77-79}$ and such neuropsychiatric consequences may be delayed ie it may be too early to judge the fallout of COVID-19 in this regard. ${ }^{18,21,79,80}$ Indirectly, the increased stress experienced by people during lockdown, may lead to hypersomnolence; either as a coping mechanism, or as part of a mental health disorder such as depression or Post-Traumatic Stress Disorder. ${ }^{81,82}$ For those who are home confined in lockdown, excessive daytime somnolence may also be a way of escaping social isolation, loneliness and/or boredom. ${ }^{30,83}$ Moreover, some people with insomnia present with excessive daytime sleepiness as their principal symptom, and may under-appreciate the effect of poor nocturnal sleep quality and/or maladaptive sleep-related behaviors in driving this. ${ }^{84}$ Finally, many patients do not differentiate between hypersomnolence, tiredness and fatigue, and may use these terms interchangeably; and/or confuse hypersomnolence with clinophilia. ${ }^{81,85}$ An appreciation of the bio-psycho-social determinants of reported hypersomnolence will be essential for clinicians in the post-lockdown era, as we are likely to face an increase in atypical sleep and neuropsychiatric presentations. $^{79}$

\section{Conclusions and Suggestions for Post-COVID Sleep Disorder's Clinical Infrastructure}

Our scientific understanding of the impact of COVID-19 continues to evolve rapidly, with a concomitant influence on the provision of clinical sleep services and new therapeutic recommendations. ${ }^{86}$ It is increasingly recognized that in the aftermath of SARS-CoV-2 infection/pandemicrelated traumatic experiences, several persistent sleep, neuropsychiatric and physical sequelae may continue long once the pandemic is over. ${ }^{1,4,8,19,30,36,79}$ In addition, due to the complexity of COVID-19 and its treatment, some affected patients may require longitudinal followup, most appropriately delivered by the interdisciplinary post-COVID-19 clinics that should ideally include sleep physicians. ${ }^{2,4,8,86}$ Such post-COVID-19 clinics should aim to provide care in a systematic and carefully coordinated fashion across multiple specialties with complementary expertise (i.e., internal medicine, psychiatry, sleep medicine, neuropsychology and physical therapy), with further accelerated access to cardiology, nephrology, infectious disease, pain specialists, pulmonary, geriatrics, neurology, nutrition, occupational health and other specialists. ${ }^{4}$ This unique design should also help facilitate exceptional teaching and international research opportunities. ${ }^{4}$ Going 
forward, shared clinical databases and open access to clinical research data, with an emphasis on educational outreach to other healthcare professionals and the wider community should be encouraged and nurtured.

These educational projects and outreaches should be preferably coordinated by international and national sleep societies, which should all work together to address the sleep difficulties incurred during lockdown, by advocating good sleep practices (e.g., minimizing pre-bed screen exposure, setting a fixed rising time, morning light exposure and exercise), ${ }^{87}$ as well as looking after mental, emotional and social health. ${ }^{88}$ Many of these recommendations have already received media attention, but await research and validation. ${ }^{85}$

Managing the sheer number of people reporting sleep difficulties, and associated health and functioning sequelae will be challenging. As we have seen with other pandemics, insomnia and associated daytime dysfunction persist long after the threat of infection has passed. ${ }^{89}$ Non-pharmacological techniques, like digital Cognitive Behavioral Therapy for Insomnia (dCBT-I) have been successful during lockdown, improving insomnia measures and daytime function. ${ }^{90}$ Further validation of the provision of these therapeutic modalities, including digital therapeutics, and an expansion to other sleep disorders, ${ }^{91}$ is now urgently required in order to stem the tsunami of lockdown-associated sleep difficulties and its consequences. Future clinical trials are similarly needed to clarify the optimal treatment strategies for sleep and other potentially long-term neuropsychiatric impacts of COVID-19. ${ }^{4}$

Finally, on the individual level, one of the rare silver linings of the pandemic is that it allowed a significant minority to take a step back and to reflect on how they live and sleep, and particularly how they work, and some of those insights have been reported as potentially lifechanging. ${ }^{68}$ These are the types of insights that can enable people to stay resilient in the face of adversity, and it will be important to try to implement these strategies into our new normal. Workplaces may need to think about adopting more flexibility in terms of working from home, or flexible work hours in industries that permit this, so that employees can better align their work and sleep with their own natural body clocks - which will make them happier and more productive in the long run.

\section{Acknowledgments}

The authors wish to thank Dr Prerna Varma and Ms Hailey Meaklim (Australia) for their leadership of the Sleep in the Time of COVID surveys, and Ms Meaklim for creating the figure for this manuscript. The authors further wish to thank all the members of the Sleep Disorders Centre at Guy's Hospital (London, UK) for all their unwavering dedication to their patients during the COVID-19 pandemic time.

\section{Disclosure}

Professor Young's independent research is funded by the National Institute for Health Research (NIHR) Biomedical Research Centre at South London and Maudsley NHS Foundation Trust and King's College London. Professor Allan H Young reports personal fees from Lundbeck, grants, personal fees, from Janssen, grants, personal fees, from Livanova, personal fees from Sunovion, grants from Compass, grants, personal fees from Novartis, personal fees from Sumitomo Dainippon Pharma, personal fees from Bionomics, personal fees from Allegan, outside the submitted work; and Employed by King's College London; Honorary Consultant SLaM (NHS UK) Deputy Editor, BJPsych Open Paid lectures and advisory boards for the following companies with drugs used in affective and related disorders: Astrazenaca, Eli Lilly, Lundbeck, Sunovion, Servier, Livanova, Janssen, Allegan, Bionomics, Sumitomo Dainippon Pharma, COMPASS Consultant to Johnson \& Johnson Consultant to Livanova Received honoraria for attending advisory boards and presenting talks at meetings organised by LivaNova. Principal Investigator in the Restore-Life VNS registry study funded by LivaNova. Principal Investigator on ESKETINTRD3004: “An Openlabel, Long-term, Safety and Efficacy Study of Intranasal Esketamine in Treatment-resistant Depression." Principal Investigator on "The Effects of Psilocybin on Cognitive Function in Healthy Participants" Principal Investigator on "The Safety and Efficacy of Psilocybin in Participants with Treatment-Resistant Depression (P-TRD)" UK Chief Investigator for Novartis MDD study MIJ821A12201 Grant funding (past and present): NIMH (USA); CIHR (Canada); NARSAD (USA); Stanley Medical Research Institute (USA); MRC (UK); Wellcome Trust (UK); Royal College of Physicians (Edin); BMA (UK); UBC-VGH Foundation (Canada); WEDC (Canada); CCS Depression Research Fund (Canada); MSFHR (Canada); NIHR (UK). Janssen (UK) No shareholdings in pharmaceutical companies.

The authors declare that the research was conducted in the absence of any commercial or financial relationships that could be construed as a potential conflict of interest. The views expressed are those of the authors and not necessarily those of the NHS, the NIHR, or the Department of Health. 


\section{References}

1. Meaklim H, Junge Moira F, Varma P, Finck WA, Jackson ML. Preexisting and post-pandemic insomnia symptoms are associated with high levels of stress, anxiety and depression globally during the COVID-19 pandemic. J Clin Sleep Med. 2021;17(10):2085-2097. doi: $10.5664 /$ jcsm. 9354

2. Rogers JP, Watson CJ, Badenoch J. et al. Neurology and neuropsychiatry of COVID-19: a systematic review and meta-analysis of the early literature reveals frequent CNS manifestations and key emerging narratives. J Neurol Neurosurg Psychiatry;2021:jnnp-2021326405. doi:10.1136/jnnp-2021-326405

3. Numbers K, Brodaty H. The effects of the COVID-19 pandemic on people with dementia. Nat Rev Neurol. 2021;17(2):69-70. doi:10.1038/s41582-020-00450-Z

4. Nakamura ZM, Nash RP, Laughon SL, Rosenstein DL. Neuropsychiatric complications of COVID-19. Curr Psychiatry Rep. 2021;23(5):25. doi:10.1007/s11920-021-01237-9

5. Nollet M, Hicks H, McCarthy AP, et al. REM sleep's unique associations with corticosterone regulation, apoptotic pathways, and behavior in chronic stress in mice. Proc Natl Acad Sci USA. 2019;116 (7):2733-2742. doi:10.1073/pnas.1816456116

6. Rosenzweig I, Glasser M, Polsek D, Leschziner GD, Williams SC, Morrell MJ. Sleep apnoea and the brain: a complex relationship. Lancet Respir Med. 2015;3(5):404-414. doi:10.1016/S22132600(15)00090-9

7. Jackson ML, Tolson J, Bartlett D, Berlowitz DJ, Varma P, Barnes M. Clinical depression in untreated obstructive sleep apnea: examining predictors and a meta-analysis of prevalence rates. Sleep Med. 2019;62:22-28. doi:10.1016/j.sleep.2019.03.011

8. Helbok R, Chou SH-Y, Beghi E, et al. NeuroCOVID: it's time to join forces globally. Lancet Neurol. 2020;19(10):805-806. doi:10.1016/ S1474-4422(20)30322-7

9. Solomon T. Neurological infection with SARS-CoV-2 - the story so far. Nat Rev Neurol. 2021;17(2):65-66. doi:10.1038/s41582-02000453-w

10. Fatima Y, Bucks RS, Mamun AA, et al. Sleep trajectories and mediators of poor sleep: findings from the longitudinal analysis of 41,094 participants of the UK Biobank cohort. Sleep Med. 2020;76:120-127. doi:10.1016/j.sleep.2020.10.020

11. Duffy, B. How the UK is Sleeping Under Lockdown [Press Release]. King's College London; 2020.

12. Perez-Carbonell L, Meurling IJ, Wassermann D, et al. Impact of the novel coronavirus (COVID-19) pandemic on sleep. J Thorac Dis. 2020;12(Suppl 2):S163-S175. doi:10.21037/jtd-cus-2020-015

13. Scarpelli S, Alfonsi V, Mangiaruga A, et al. Pandemic nightmares: effects on dream activity of the COVID-19 lockdown in Italy. $J$ Sleep Res. 2021;30(5):e13300. doi:10.1111/jsr.13300

14. Barrett D. Dreams about COVID-19 versus normative dreams: trends by gender. Dreaming. 2020;30(3):216-221. doi:10.1037/drm0000149

15. Scarpelli S, Gorgoni M, Alfonsi V, et al. The impact of the end of COVID confinement on pandemic dreams, as assessed by a weekly sleep diary: a longitudinal investigation in Italy. J Sleep Res. 2021: e13429. doi:10.1111/jsr.13429

16. Dzierzewski JM, Dautovich ND, Ravyts SG, Perez E, Soto P, Donovan EK. Insomnia symptoms during the COVID-19 pandemic: an examination of biopsychosocial moderators. Sleep Med. 2021. doi:10.1016/j.sleep.2021.02.018

17. Florea C, Topalidis P, Hauser T, et al. Sleep during COVID-19 lockdown: a cross-cultural study investigating job system relevance. Biochem Pharmacol. 2021;191:114463. doi:10.1016/j.bcp.2021.114463

18. Rosenzweig I, Mitrečić D, Petanjek Z, et al. Does damage to hypothalamic paraventricular nucleus underlie symptoms of ultradian rhythm disorder and an increased anxiety in coronavirus disease 2019? Croat Med J. 2020;61(4):377-380. doi:10.3325/ cmj.2020.61.377
19. Steenblock C, Todorov V, Kanczkowski W, et al. Severe acute respiratory syndrome coronavirus 2 (SARS-CoV-2) and the neuroendocrine stress axis. Mol Psychiatry. 2020;25(8):1611-1617. doi:10.1038/s41380-020-0758-9

20. Goldstein AN, Walker MP. The role of sleep in emotional brain function. Annu Rev Clin Psychol. 2014;10(1):679-708. doi:10.1146/ annurev-clinpsy-032813-153716

21. Suzuki YJ, Gychka SG. SARS-CoV-2 spike protein elicits cell signaling in human host cells: implications for possible consequences of COVID-19 vaccines. Vaccines (Basel). 2021;9(1):36.

22. Garrigues E, Janvier P, Kherabi Y, et al. Post-discharge persistent symptoms and health-related quality of life after hospitalization for COVID-19. $J$ Infect. 2020;81(6):e4-e6. doi:10.1016/j.jinf.2020. 08.029

23. Taquet M, Geddes JR, Husain M, Luciano S, Harrison PJ. 6-month neurological and psychiatric outcomes in 236379 survivors of COVID-19: a retrospective cohort study using electronic health records. Lancet Psychiatry. 2021;8(5):416-427. doi:10.1016/S22150366(21)00084-5

24. Rathod S, Pallikadavath S, Young AH, et al. Psychological impact of COVID-19 pandemic: protocol and results of first three weeks from an international cross-section survey - focus on health professionals. $J$ Affect Disord Rep. 2020;1:100005. doi:10.1016/j.jadr.2020.100005

25. Suzuki YJ, Nikolaienko SI, Dibrova VA, et al. SARS-CoV-2 spike protein-mediated cell signaling in lung vascular cells. Vascul Pharmacol. 2021;137:106823. doi:10.1016/j.vph.2020.106823

26. Suryawanshi RK, Koganti R, Agelidis A, Patil CD, Shukla D. Dysregulation of cell signaling by SARS-CoV-2. Trends Microbiol. 2021;29(3):224-237. doi:10.1016/j.tim.2020.12.007

27. Domazet-Lošo T. mRNA Vaccines: Why is the Biology of Retroposition Ignored?

28. Galli F, Reglero G, Bartolini D, Visioli F. Better prepare for the next one. Lifestyle lessons from the COVID-19 pandemic. PharmaNutrition. 2020;12:100193. doi:10.1016/j.phanu.2020.100193

29. Altena E, Baglioni C, Espie CA, et al. Dealing with sleep problems during home confinement due to the COVID-19 outbreak: practical recommendations from a task force of the European CBT-I Academy. J Sleep Res. 2020;29(4):e13052. doi:10.1111/jsr.13052

30. Gupta R, Grover S, Basu A, et al. Changes in sleep pattern and sleep quality during COVID-19 lockdown. Indian J Psychiatry. 2020;62 (4):370-378. doi:10.4103/psychiatry.IndianJPsychiatry_523_20

31. Kearsley R, Duffy CC. The COVID-19 information pandemic: how have we managed the surge? Anaesthesia. 2020;75(8):993-996. doi:10.1111/anae.15121

32. Leger D, Beck F, Fressard L, Verger P, Peretti-Watel P; Group C. Poor sleep associated with overuse of media during the COVID-19 lockdown. Sleep. 2020;43(10). doi:10.1093/sleep/zsaa125

33. Simpson N, Manber R. Treating Insomnia during the COVID-19 pandemic: observations and perspectives from a behavioral sleep medicine clinic. Behav Sleep Med. 2020;18(4):573-575. doi:10.1080/15402002.2020.1765781

34. Blume C, Schmidt MH, Cajochen C. Effects of the COVID-19 lockdown on human sleep and rest-activity rhythms. Curr Biol. 2020;30 (14):R795-R797.

35. Wright KP, Linton SK, Withrow D, et al. Sleep in University students prior to and during COVID-19 stay-at-home orders. Curr Biol. 2020;30(14):R797-R798. doi:10.1016/j.cub.2020.06.022

36. Robbins R, Affouf M, Weaver MD, et al. Estimated sleep duration before and during the COVID-19 pandemic in major metropolitan areas on different continents: observational study of smartphone app data. J Med Internet Res. 2021;23(2):e20546. doi:10.2196/20546

37. Beck F, Leger D, Cortaredona S, Verger P, Peretti-Watel P; group C. Would we recover better sleep at the end of Covid-19? A relative improvement observed at the population level with the end of the lockdown in France. Sleep Med. 2021;78:115-119. doi:10.1016/j. sleep.2020.11.029 
38. Salfi F, Lauriola M, D'Atri A, et al. Demographic, psychological, chronobiological, and work-related predictors of sleep disturbances during the COVID-19 lockdown in Italy. Sci Rep. 2021;11(1):11416. doi:10.1038/s41598-021-90993-y

39. Staller N, Randler C. Changes in sleep schedule and chronotype due to COVID-19 restrictions and home office. Somnologie (Berl). 2020;25:131-137.

40. Varma P, Burge M, Meaklim H, Junge M, Jackson ML. Poor sleep quality and its relationship with individual characteristics, personal experiences and mental health during the COVID-19 pandemic. Int J Environ Res Public Health. 2021;18(11):11. doi:10.3390/ ijerph18116030

41. Phillips AJK, Clerx WM, O'Brien CS, et al. Irregular sleep/wake patterns are associated with poorer academic performance and delayed circadian and sleep/wake timing. Sci Rep. 2017;7(1):3216. doi:10.1038/s41598-017-03171-4

42. Huang T, Mariani S, Redline S. Sleep irregularity and risk of cardiovascular events. J Am Coll Cardiol. 2020;75(9):991-999. doi:10.1016/j.jacc.2019.12.054

43. Cellini N, Canale N, Mioni G, Costa S. Changes in sleep pattern, sense of time and digital media use during COVID-19 lockdown in Italy. J Sleep Res. 2020;29(4):e13074. doi:10.1111/jsr.13074

44. Zhao X, Lan M, Li H, Yang J. Perceived stress and sleep quality among the non-diseased general public in China during the 2019 coronavirus disease: a moderated mediation model. Sleep Med. 2021;77:339-345. doi:10.1016/j.sleep.2020.05.021

45. Franceschini C, Musetti A, Zenesini C, et al. Poor sleep quality and its consequences on mental health during the COVID-19 lockdown in Italy. Front Psychol. 2020;11:574475. doi:10.3389/ fpsyg.2020.574475

46. Sultana A, Tasnim S, Hossain MM, Bhattacharya S, Purohit N. Digital screen time during the COVID-19 pandemic: a public health concern. F1000Research. 2021;10:81. doi:10.12688/ f1000research.50880.1

47. Smith L, Jacob L, Trott M, et al. The association between screen time and mental health during COVID-19: a cross sectional study. Psychiatry Res. 2020;292:113333. doi:10.1016/j. psychres.2020.113333

48. Salfi F, Amicucci G, Corigliano D, et al. Changes of evening exposure to electronic devices during the COVID-19 lockdown affect the time course of sleep disturbances. Sleep. 2021;44(9). doi:10.1093/ sleep/zsab080.

49. Voitsidis P, Gliatas I, Bairachtari V, et al. Insomnia during the COVID-19 pandemic in a Greek population. Psychiatry Res. 2020;289:113076. doi:10.1016/j.psychres.2020.113076

50. Nedergaard M, Goldman SA. Glymphatic failure as a final common pathway to dementia. Science. 2020;370(6512):50-56. doi:10.1126/ science.abb8739

51. Borbely AA, Daan S, Wirz-Justice A, Deboer T. The two-process model of sleep regulation: a reappraisal. J Sleep Res. 2016;25 (2):131-143. doi:10.1111/jsr.12371

52. Đogaš Z, Lušić Kalcina L, Pavlinac Dodig I, et al. The effect of COVID-19 lockdown on lifestyle and mood in Croatian general population: a cross-sectional study. Croat Med J. 2020;61 (4):309-318. doi:10.3325/cmj.2020.61.309

53. Czeisler MÉ, Wiley JF, Facer-Childs ER, et al. Mental health, substance use, and suicidal ideation during a prolonged COVID-19related lockdown in a region with low SARS-CoV-2 prevalence. J Psychiatr Res. 2021;140:533-544. doi:10.1016/j. jpsychires.2021.05.080

54. Abdool Karim SS. The South African response to the pandemic. N Engl J Med. 2020;382(24):e95. doi:10.1056/NEJMc2014960

55. Cellini N, Conte F, De Rosa O, et al. Changes in sleep timing and subjective sleep quality during the COVID-19 lockdown in Italy and Belgium: age, gender and working status as modulating factors. Sleep Med. 2021;77:112-119. doi:10.1016/j.sleep.2020.11.027
56. Czeisler MÉ, Lane RI, Petrosky E, et al. Mental health, substance use, and suicidal ideation during the COVID-19 pandemic - United States, June 24- 30, 2020. MMWR Morb Mortal Wkly Rep. 2020;69 (32):1049-1057. doi:10.15585/mmwr.mm6932a1

57. Rossell SL, Neill E, Phillipou A, et al. An overview of current mental health in the general population of Australia during the COVID-19 pandemic: results from the COLLATE project. Psychiatry Res. 2021;296:113660. doi:10.1016/j.psychres.2020.113660

58. Salfi F, Lauriola M, Amicucci G, et al. Gender-related time course of sleep disturbances and psychological symptoms during the COVID-19 lockdown: a longitudinal study on the Italian population. Neurobiol Stress. 2020;13:100259. doi:10.1016/j.ynstr.2020.100259

59. Matias T, Dominski FH, Marks DF. Human needs in COVID-19 isolation. $J$ Health Psychol. 2020;25(7):871-882. doi:10.1177/ 1359105320925149

60. Baron KG, Culnan E. Sleep and Healthy Decision Making. Academic Press; 2019:359-369.

61. Ramalho R, Adiukwu F, Gashi Bytyci D, et al. Alcohol and tobacco use during the COVID-19 pandemic. A call for local actions for global impact. Front Psychiatry. 2021;12:634254. doi:10.3389/ fpsyt.2021.634254

62. Doraiswamy S, Cheema S, Al Mulla A, Mamtani R. COVID-19 lockdown and lifestyles - a narrative review. Med Pharmacol. 2021. doi:10.20944/preprints202011.0532.v2

63. Stein MD, Friedmann PD. Disturbed sleep and its relationship to alcohol use. Subst Abus. 2005;26(1):1-13. doi:10.1300/J465v26n01_01

64. Kudumija Slijepcevic M, Jukic V, Novalic D, Zarkovic-Palijan T, Milosevic M, Rosenzweig I. Alcohol abuse as the strongest risk factor for violent offending in patients with paranoid schizophrenia. Croat Med J. 2014;55(2):156-162. doi:10.3325/cmj.2014.55.156

65. Bu F, Steptoe A, Fancourt D. 2020.

66. Chaudhury S, Samudra M. COVID-19 lockdown: psychological effects. Med J Dr DY Patil Vidyapeeth. 2020;13(6):580. doi:10.4103/mjdrdypu.mjdrdypu_230_20

67. Cacioppo JT, Hawkley LC, Berntson GG, et al. Do lonely days invade the nights? Potential social modulation of sleep efficiency. Psychol Sci. 2002;13(4):384-387. doi:10.1111/j.09567976.2002.00469.x

68. Rosenzweig I. Vignette on Canetti, crowds, and the self in times of COVID-19. Croat Med J. 2020;61(4):307-308. doi:10.3325/ cmj.2020.61.307

69. Acharibasam JW, Chireh B, Menegesha HG. Assessing anxiety, depression and insomnia symptoms among Ebola survivors in Africa: a meta-analysis. PLoS One. 2021;16(2):e0246515. doi:10.1371/journal. pone. 0246515

70. Pieh C, Budimir S, Delgadillo J, Barkham M, Fontaine JRJ, Probst T. Mental health during COVID-19 lockdown in the United Kingdom. Psychosom Med. 2021;83(4):328-337. doi:10.1097/PSY.00000 00000000871

71. Alimoradi Z, Broström A, Tsang HWH, et al. Sleep problems during COVID-19 pandemic and its' association to psychological distress: a systematic review and meta-analysis. EClinicalMedicine. 2021;36:100916. doi:10.1016/j.eclinm.2021.100916

72. Kocevska D, Blanken TF, Van Someren EJW, Rösler L. Sleep quality during the COVID-19 pandemic: not one size fits all. Sleep Med. 2020;76:86-88. doi:10.1016/j.sleep.2020.09.029

73. Ferri P, Guadi M, Marcheselli L, Balduzzi S, Magnani D, Di Lorenzo R. The impact of shift work on the psychological and physical health of nurses in a general hospital: a comparison between rotating night shifts and day shifts. Risk Manag Healthc Policy. 2016;9:203-211. doi:10.2147/RMHP.S115326

74. Mohanty A, Kabi A, Mohanty AP. Health problems in healthcare workers: a review. J Family Med Prim Care. 2019;8(8):2568-2572.

75. Fatima Y, Bucks RS, Mamun AA, et al. Shift work is associated with increased risk of COVID-19: findings from the UK Biobank cohort. J Sleep Res. 2021;30:e13326. 
76. Blume C, Schmidt MH, Cajochen C. Effects of the COVID-19 lockdown on human sleep and rest-activity rhythms. Curr Biol. 2020;30 (14):R795-R797.

77. Manjunatha N, Math SB, Kulkarni GB, Chaturvedi SK. The neuropsychiatric aspects of influenza/swine flu: a selective review. Ind Psychiatry J. 2011;20(2):83-90. doi:10.4103/09726748.102479

78. Economo CV. Encephalitis lethargica, its sequelae and treatment. J Am Med Assoc. 1932;98(3):255. doi:10.1001/jama.1932.0273 0290071039

79. Troyer EA, Kohn JN, Hong S. Are we facing a crashing wave of neuropsychiatric sequelae of COVID-19? Neuropsychiatric symptoms and potential immunologic mechanisms. Brain Behav Immun. 2020;87:34-39. doi:10.1016/j.bbi.2020.04.027

80. Salfi F, D'Atri A, Tempesta D, Ferrara M. Sleeping under the waves: a longitudinal study across the contagion peaks of the COVID-19 pandemic in Italy. J Sleep Res. 2021;30(5):e13313. doi:10.1111/ jsr. 13313

81. Barateau L, Lopez R, Franchi JA, Dauvilliers Y. Hypersomnolence, hypersomnia, and mood disorders. Curr Psychiatry Rep. 2017;19 (2):13. doi:10.1007/s11920-017-0763-0

82. Selsick H, O'Regan D. Sleep disorders in psychiatry. BJ Psych Advan. 2018;24(4):273-283. doi:10.1192/bja.2018.8

83. Duggan KA, McDevitt EA, Whitehurst LN, Mednick SC. To nap, perchance to DREAM: a factor analysis of college students' selfreported reasons for napping. Behav Sleep Med. 2018;16(2):135-153. doi:10.1080/15402002.2016.1178115

84. Lichstein KL, Wilson NM, Noe SL, Aguillard RN, Bellur SN. Daytime sleepiness in insomnia: behavioral, biological and subjective indices. Sleep. 1994;17(8):693-702. doi:10.1093/sleep/17.8.693

85. Becker PM. Overview of sleep management during COVID-19. Sleep Med. 2021. doi:10.1016/j.sleep.2021.04.024
86. Jauhar S, Lai S, Bonoldi I, et al. Early intervention in psychosis during the COVID-19 pandemic: Maudsley recommendations. Eur Neuropsychopharmacol. 2021;47:130-135. doi:10.1016/j.euroneuro. 2021.02.005

87. Morin CM, Carrier J, Bastien C, Godbout R, Canadian S, Circadian N. Sleep and circadian rhythm in response to the COVID-19 pandemic. Can J Public Health. 2020;111(5):654-657. doi:10.17269/s41997-020-00382-7

88. Datta K, Tripathi M. Sleep and Covid-19. Neurol India. 2021;69 (1):26-31. doi:10.4103/0028-3886.310073

89. Magill E, Siegel Z, Pike KM. The mental health of frontline health care providers during pandemics: a rapid review of the literature. Psychiatr Serv. 2020;71(12):1260-1269. doi:10.1176/appi. ps.202000274

90. Arnedt JT, Conroy DA, Mooney A, Furgal A, Sen A, Eisenberg D. Telemedicine versus face-to-face delivery of cognitive behavioral therapy for insomnia: a randomized controlled noninferiority trial. Sleep. 2021;44(1). doi:10.1093/sleep/zsaa136

91. O'Regan D, Nesbitt A, Biabani N, et al. A novel group cognitive behavioural therapy approach to adult non-rapid eye movement parasomnias. Front Psychiatry. doi:10.3389/fpsyt.2021.679272

92. Calhoon GG, Tye KM. Resolving the neural circuits of anxiety. Nat Neurosci. 2015;18(10):1394-1404. doi:10.1038/nn.4101

93. Mukerjee S, Gao H, Xu J, Sato R, Zsombok A, Lazartigues E. ACE2 and ADAM17 interaction regulates the activity of presympathetic neurons. Hypertension. 2019;74(5):1181-1191. doi:10.1161/ HYPERTENSIONAHA.119.13133

94. Ferguson AV, Latchford KJ, Samson WK. The paraventricular nucleus of the hypothalamus - a potential target for integrative treatment of autonomic dysfunction. Expert Opin Ther Targets. 2008;12 (6):717-727. doi:10.1517/14728222.12.6.717
Nature and Science of Sleep

\section{Publish your work in this journal}

Nature and Science of Sleep is an international, peer-reviewed, open access journal covering all aspects of sleep science and sleep medicine, including the neurophysiology and functions of sleep, the genetics of sleep, sleep and society, biological rhythms, dreaming, sleep disorders and therapy, and strategies to optimize healthy sleep.
The manuscript management system is completely online and includes a very quick and fair peer-review system, which is all easy to use. Visit http://www.dovepress.com/testimonials.php to read real quotes from published authors. 